Talk presented at the QCD Euroconference 96, Montpellier (France) July 4-12, 1996.

PITHA $96 / 3 Q$

September 1996

\title{
EXPERIMENTAL TESTS OF ASYMPTOTIC FREEDOM
}

\author{
Siegfried Bethke ${ }^{\mathrm{a}}$ \\ ${ }^{a} I I I$. Physikalisches Institut, RWTH, D - 52056 Aachen, Germany
}

\begin{abstract}
Measurements which probe the energy dependence of $\alpha_{\mathrm{s}}$, the coupling strength of the strong interaction, are reviewed. Jet counting in $\mathrm{e}^{+} \mathrm{e}^{-}$annihilation, combining results obtained in the centre of mass energy range from 22 to $133 \mathrm{GeV}$, provides direct evidence for an asymptotically free coupling, without the need to determine explicit values of $\alpha_{\mathrm{s}}$. Recent results from jet production in $e p$ and in $p \bar{p}$ collisions, obtained in single experiments spanning large ranges of momentum transfer, $Q^{2}$, are in good agreement with the running of $\alpha_{\mathrm{s}}$ as predicted by QCD. Mass spectra of hadronic decays of $\tau$-leptons are analysed to probe the running $\alpha_{\mathrm{s}}$ in the very low energy domain, $0.7 \mathrm{GeV}^{2}<Q^{2}<M_{\tau}^{2}$. An update of the world summary of measurements of $\alpha_{\mathrm{s}}\left(Q^{2}\right)$ consistently proves the energy dependence of $\alpha_{\mathrm{s}}$ and results in a combined average of $\alpha_{\mathrm{s}}\left(M_{\mathrm{Z}^{0}}\right)=0.118 \pm 0.006$.
\end{abstract}

\section{INTRODUCTION}

Since Quantum Chromodynamics [1] and the concept of asymptotic freedom [2] were introduced to describe the dynamics of hadronic processes at high momentum transfers, several "key" predictions of the theory were successfully tested by experiment: 'Evidence for jet structure in hadron production by $\mathrm{e}^{+} \mathrm{e}^{-}$annihilation' was found in 1975 [3], the gluon was explicitly observed at the PETRA $\mathrm{e}^{+} \mathrm{e}^{-}$storage ring in 1979 [4. The first measurement of the coupling strength $\alpha_{\mathrm{s}}$, the basic free parameter of the theory, was reported in that same year [5], based on leading order perturbative QCD. The first determination of $\alpha_{\mathrm{s}}$ in next-to-leading order (NLO) QCD dates back to 1982 [6].

After these pioneering years, many further tests of QCD were performed in $\mathrm{e}^{+} \mathrm{e}^{-}$annihilation, in deep inelastic lepton-nucleon scattering and at hadron colliders. In 1988, first evidence for the running $\alpha_{\mathrm{s}}$ was obtained from the energy dependence of 3-jet event production rates in $\mathrm{e}^{+} \mathrm{e}^{-}$annihilation [7]. An update and a summary of these measurements will be presented in Section 3 of this review.

Although many determinations of $\alpha_{\mathrm{s}}$, in the energy range of $Q \sim 4$ to $46 \mathrm{GeV}$, were available by 1990, the running of $\alpha_{\mathrm{s}}$ could not convincingly be seen from those results $[8$. Only in 1992, a compilation of measurements of $\alpha_{\mathrm{s}}$ in the energy range from $1.78 \mathrm{GeV}$ (the mass of the $\tau$ lepton) to $91.2 \mathrm{GeV}$ (the mass of the $\mathrm{Z}^{0}$-boson), could demonstrate the characteristic energy dependence of the strong coupling [9].

The actual evidence for the running coupling strength or, equivalently, for asymptotic freedom is summarised in this review. The results from jet production rates in $\mathrm{e}^{+} \mathrm{e}^{-}$annihilation are presented in Section 3. Recent studies of jet production and of the proton structure function $F_{2}$ in deep inelastic electron-proton collisions are discussed in Section 4. New results from jets in $p \bar{p}$ collisions and from hadronic decays of $\tau$-leptons, demonstrating the energy dependence of $\alpha_{\mathrm{s}}$ in ranges of very high and very low momentum transfers, respectively, are presented in Sections 5 and 6. An update of the world summary of $\alpha_{\mathrm{s}}$ measurements is finally given in Section 7 .

This report is restricted to results which were published at the time of this conference; preliminary results are not taken into account.

\section{QCD AND THE RUNNING $\alpha_{\mathrm{s}}$}

Within perturbative QCD, the energy dependence of $\alpha_{\mathrm{s}}$ is given by the $\beta$-function:

$$
\begin{aligned}
\mu \frac{\partial \alpha_{\mathrm{s}}}{\partial \mu} & =-\frac{\beta_{0}}{2 \pi} \alpha_{\mathrm{s}}^{2}-\frac{\beta_{1}}{4 \pi^{2}} \alpha_{\mathrm{s}}^{3}-\frac{\beta_{2}}{64 \pi^{3}} \alpha_{\mathrm{s}}^{4}-\ldots \\
\beta_{0} & =11-\frac{2}{3} N_{f}
\end{aligned}
$$




$$
\begin{aligned}
& \beta_{1}=51-\frac{19}{3} N_{f} \\
& \beta_{2}=2857-\frac{5033}{9} N_{f}+\frac{325}{27} N_{f}^{2},
\end{aligned}
$$

where $N_{f}$ is the number of quark flavours with masses less than the energy scale $\mu$. A solution of Equation 1, in third order expansion, is 10]

$$
\begin{aligned}
\alpha_{s}(\mu) & =\frac{4 \pi}{\beta_{0} \ln (\mu / \Lambda)^{2}}\left[1-2 \frac{\beta_{1}}{\beta_{0}^{2}} \frac{\ln \left(\ln (\mu / \Lambda)^{2}\right)}{\ln (\mu / \Lambda)^{2}}\right. \\
& +\frac{4 \beta_{1}^{2}}{\beta_{0}^{4} \ln ^{2}(\mu / \Lambda)^{2}}\left(\left(\ln \left[\ln (\mu / \Lambda)^{2}\right]-\frac{1}{2}\right)^{2}\right. \\
& \left.\left.+\frac{\beta_{2} \beta_{0}}{8 \beta_{1}^{2}}-\frac{5}{4}\right)\right] .
\end{aligned}
$$

At large energy scales $\mu$, or equivalently at small distances, $\alpha_{\mathrm{s}}$ vanishes logarithmically; this behaviour of $\alpha_{\mathrm{s}}$ is called 'asymptotic freedom'.

In this report all calculations, equations and results refer to the 'modified minimal subtraction scheme' $(\overline{\mathrm{MS}})$ [11. More detailed information about the basic QCD equations and for the treatment of heavy quark flavour thresholds can be found e.g. in 1013 .

\section{JET RATES IN $\mathrm{e}^{+} \mathrm{e}^{-}$ANNIHILATION}

Studies of hadron jets provide the most intuitive tests of the underlying parton (i.e. quark and gluon) structure of hadronic events. The most commonly used algorithm to reconstruct jets in $\mathrm{e}^{+} \mathrm{e}^{-}$annihilation was introduced by the JADE collaboration [7]: the scaled pair mass of any two resolvable jets $i$ and $j$ in a hadronic event, $y_{i j}=M_{i j}^{2} / E_{\mathrm{vis}}^{2}$, is required to exceed a threshold value $y_{\text {cut }}$, where $E_{\text {vis }}$ is the total visible (measured) energy of the event. In a recursive process, the pair of particles or clusters of particles which has the smallest value of $y_{i j}$ is replaced by (or 'recombined' into) a single jet $k$ with fourmomentum $p_{k}=p_{i}+p_{j}$, as long as $y_{i j}<y_{\text {cut }}$. The procedure is repeated until all $y_{i j}$ are larger than the jet resolution parameter $y_{\text {cut }}$, and the remaining clusters of particles are called jets.

Several methods of jet recombination and definitions of $M_{i j}$ exist, for which QCD predictions in complete $\mathcal{O}\left(\alpha_{\mathrm{s}}^{2}\right)$ perturbation theory [14], based on the matrix elements of Ellis, Ross and Terrano [15], are available. In $\mathcal{O}\left(\alpha_{\mathrm{s}}^{2}\right)$, the relative 2-,

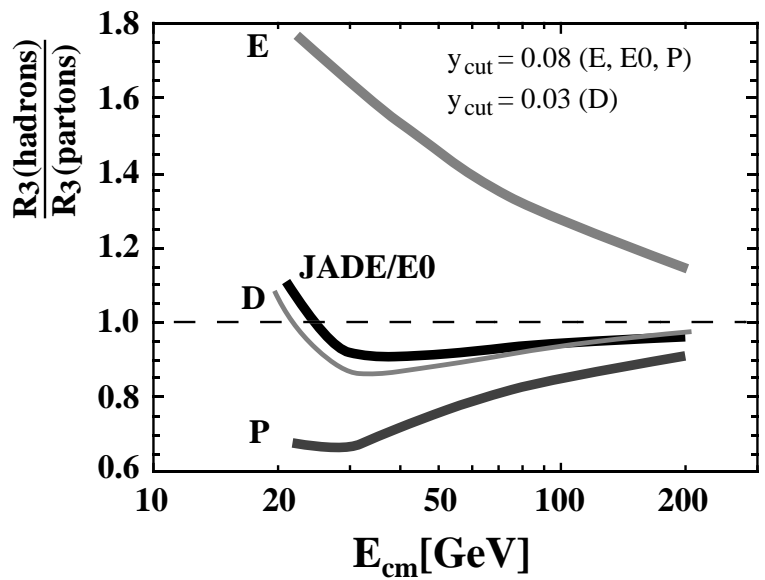

Figure 1. The ratio $r$ of 3-jet event rates, calculated from JETSET QCD shower model events before and after hadronisation, as a function of $E_{c m}$, for different jet algorithms.

3 - and 4-jet production rates, $R_{n}=\sigma_{\mathrm{n}-\text { jet }} / \sigma_{\text {tot }}$, where $\sigma_{\text {tot }}$ is the total hadronic cross section and $\sigma_{\mathrm{n}-\text { jet }}$ are the cross sections for $n$-parton event production, are quadratic functions of the running coupling constant $\alpha_{\mathrm{s}}(\mu)$. In particular,

$R_{3}\left(y_{c}, \mu\right)=A\left(y_{c}\right) \frac{\alpha_{\mathrm{s}}(\mu)}{2 \pi}+B\left(y_{c}, x_{\mu}\right)\left(\frac{\alpha_{\mathrm{s}}(\mu)}{2 \pi}\right)^{2}$,

where $\mu=x_{\mu} E_{c m}$ is the renormalisation scale at which $\alpha_{\mathrm{s}}$ is evaluated, $x_{\mu}$ is the renormalisation scale factor and $y_{c} \equiv y_{\text {cut }}$.

The energy dependence of $R_{3}$ is only determined by the running $\alpha_{\mathrm{s}}$; the scale factor $x_{\mu}$ although it's optimal value is not given by the theory - is not expected to change with energy. Jet production rates are therefore an ideal tool to test the energy dependence of $\alpha_{\mathrm{s}}$, without the need to actually determine $\alpha_{\mathrm{s}}$ itself.

The first analysis in this sense was done by JADE [7]. The original JADE (also called E0) scheme with $M_{i j}^{2}=2 E_{i} E_{j}\left(1-\cos \theta_{i j}\right)$, where $E_{i}$ and $E_{j}$ are the energies of the particles and $\theta_{i j}$ is the angle between them, has the smallest hadronisation corrections with only a weak dependence on the centre of mass energy, $E_{c m}$. This is demonstrated in Figure 1, where the ratio $r=R_{3}$ (hadrons) / $R_{3}$ (partons) predicted by the JETSET QCD shower model [16] is plotted 


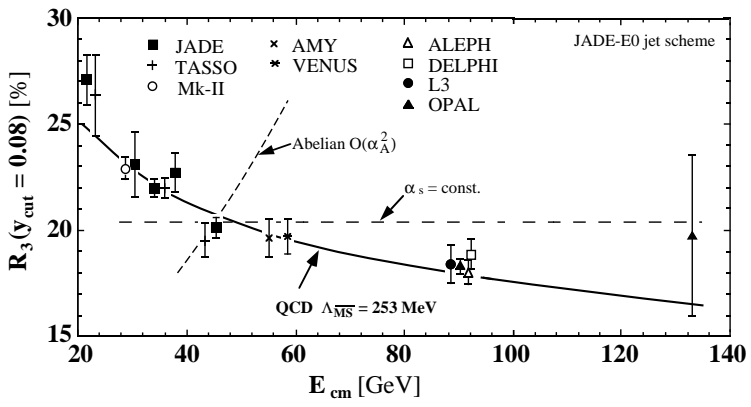

Figure 2. Energy dependence of three-jet event production rates $R_{3}$, using the JADE E0 jet scheme with $y_{\text {cut }}=0.08$. The measurements are compared with predictions of analytic $\mathcal{O}\left(\alpha_{\mathrm{s}}^{2}\right)$ QCD calculations, with the hypothesis of an energy independent $\alpha_{\mathrm{s}}$ and with the abelian vector theory in $\mathcal{O}\left(\alpha_{A}^{2}\right)$.

as a function of $E_{c m}$, for constant values of $y_{\text {cut }}$ 17. For all jet algorithms, the quantity $(1-r)$ shows an approximate $1 / E_{c m}$ behaviour at large $E_{c m}$, as expected for non-perturbative hadronisation effects. At smaller energies, usually for $\sqrt{y_{\text {cut }}} E_{c m}<7 \mathrm{GeV}, r$ increases with decreasing $E_{c m}$ because of misassignments of jets, caused by hadronisation fluctuations and heavy quark decays. For the JADE E0 algorithm, $|1-r|$ is small enough and the energy dependence of $r$ is sufficiently flat for $E_{c m}$ between 25 and $200 \mathrm{GeV}$ to be approximated by a constant within a systematic uncertainty of $\pm 2 \%$. This feature makes an important impact on the experimental evidence for asymptotic freedom.

A compilation of the experimental results of $R_{3}$, analysed with the JADE (E0) jet finder at different $E_{c m}$ using $y_{\text {cut }}=0.08$, is presented in Fig. 2 [7, 18, 19]. The data are compared with fit results of analytic $\mathcal{O}\left(\alpha_{\mathrm{s}}^{2}\right)$ QCD calculations [14], of the hypothesis of an energy independent coupling constant and of the abelian, QED-like vector theory in $O\left(\alpha_{A}^{2}\right)$, where $\alpha_{A}$ was adjusted such that the jet rates at $E_{c m}=44 \mathrm{GeV}$ are reproduced 17.

For the QCD predictions and the hypothesis of $\alpha_{\mathrm{s}}=$ constant, the free parameters $\Lambda_{\overline{M S}}$ and a constant 3-jet rate $\left\langle R_{3}\right\rangle$, respectively, were determined by minimising $\chi^{2}$ for the data from $E_{c m}$ $=29$ to $133 \mathrm{GeV}$. The data points at $E_{c m}=22$

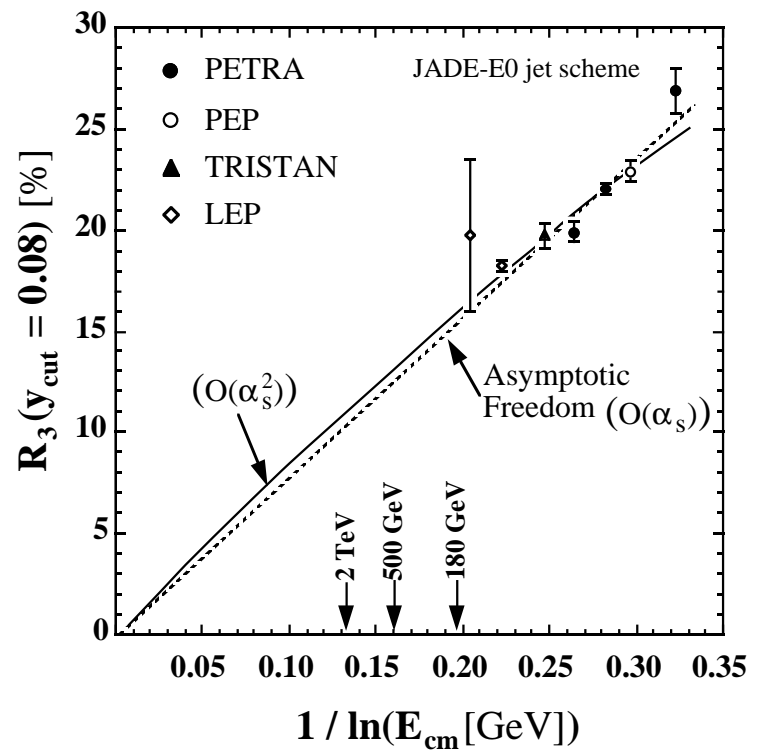

Figure 3. The same data as shown in Fig.2, combined at similar energies, now as a function of $1 / \ln \left(E_{c m}\right)$.

$\mathrm{GeV}$ were not included in the fit since hadronisation effects may already bias the measurements at this energy, see Fig. 1.

For QCD, the fit results in $\Lambda_{\overline{M S}}=(253 \pm$ $12 \mathrm{MeV})$, which corresponds to $\alpha_{\mathrm{s}}\left(M_{\mathrm{Z}^{0}}\right)=$ $0.120 \pm 0.001$ (stat. error only), and $\chi^{2}=8.1$ for 13 degrees of freedom 7 , corresponding to a confidence level (CL) of $84 \%$. A linear fit through the data (not shown in Fig. 2) gives $\chi^{2}=12.7$ for 12 degrees of freedom (CL $=39 \%$ ). The hypothesis of $\alpha_{\mathrm{s}}=$ constant with $\chi^{2}=72\left(\mathrm{CL}=3.4 \times 10^{-10}\right)$ and the abelian theory, the $\chi^{2}$ of which tends to infinity, are entirely ruled out by the data.

The experimental evidence for asymptotic freedom is further demonstrated in Fig. 3, where the same experimental data, however combined at similar c.m. energies, are plotted as a function of $1 / \ln \left(E_{c m}\right)$. The dashed line is a fit to the leading order QCD prediction, namely $R_{3} \propto$ $\alpha_{\mathrm{s}} \propto 1 / \ln E_{c m}$. The corresponding prediction in $\mathcal{O}\left(\alpha_{\mathrm{s}}^{2}\right)$ is also shown, indicating that higher order

* In order to account for the small, energy dependent hadronisation effects as predicted by the model calculations shown in Fig. 1, a relative systematic point-to-point uncertainty of $\pm 2 \%$ is included when calculating $\chi^{2}$. 
terms affect the energy dependence of $R_{3}$ only slightly. At infinite energies $\left(1 / \ln \left(E_{c m}\right) \rightarrow 0\right)$, $R_{3}$ and $\alpha_{\mathrm{s}}$ are expected to vanish; an assumption which is in good agreement by the data.

While the most recent data from LEP-1.5 $\left(E_{c m} \sim 133 \mathrm{GeV}\right)$ are statistically very limited, which will most likely not be improved in the future, it is expected that LEP-2 $\left(E_{c m} \sim 175 \mathrm{GeV}\right)$ will provide another data point with an absolute error of $\Delta\left(R_{3}\right) \sim 1 \%$. The significance of data from future high energy $\mathrm{e}^{+} \mathrm{e}^{-}$linear colliders can be inferred from Figure 3: at $E_{c m}=500 \mathrm{GeV}$, the statistical error $\Delta\left(R_{3}\right)$ for 1000 hadronic events will be about $1 \%$. From the point of view of the previous experiments at PETRA and PEP and with the eyes of $\mathrm{QCD}$, i.e. on a logarithmic energy scale, a linear collider at $E_{c m}=500 \mathrm{GeV}$ is almost half-way to infinte energies!

\section{RUNNING $\alpha_{\mathrm{s}}$ FROM e-p COLLISIONS}

In deep inelastic electron-proton scattering (DIS), hadronic final states can be studied in a wide range of energy scales $Q^{2}$, the squared four-momentum transfer from the incident lepton. Both experiments at HERA, H1 and ZEUS, determined $\alpha_{\mathrm{s}}\left(Q^{2}\right)$ from jet rates measured in the energy range of $10 \mathrm{GeV}^{2}<Q^{2}<4000 \mathrm{GeV}^{2}$ [20,21], using a modified JADE jet algorithm where the proton remnant is treated as a pseudoparticle which carries only longitudinal momentum (i.e. along the beam direction).

The jet resolution parameter is given by $y_{c}=$ $M_{i j}^{2} / W^{2}$, where $W$ is the invariant mass of the hadronic system and $M_{i j}$ the invariant pair masses of all objects used including the pseudoparticle. This choice of algorithm ensures a jet classification which is similar to that used in $\mathrm{e}^{+} \mathrm{e}^{-}$ annihilation. The jet production rates $R_{N+1}$ are calculated from the number of events with $N$ resolvable jets inside the acceptance region, where " +1 " denotes the pseudoparticle (or pseudojet) from the proton remnant.

In DIS, jet rates by themselves cannot demonstrate the running of the coupling since theory predicts that the QCD coefficients of $\alpha_{\mathrm{s}}$ also depend, in contrast to the case of $\mathrm{e}^{+} \mathrm{e}^{-}$annihilation (see Eq. 3), on $Q$ through the parton density

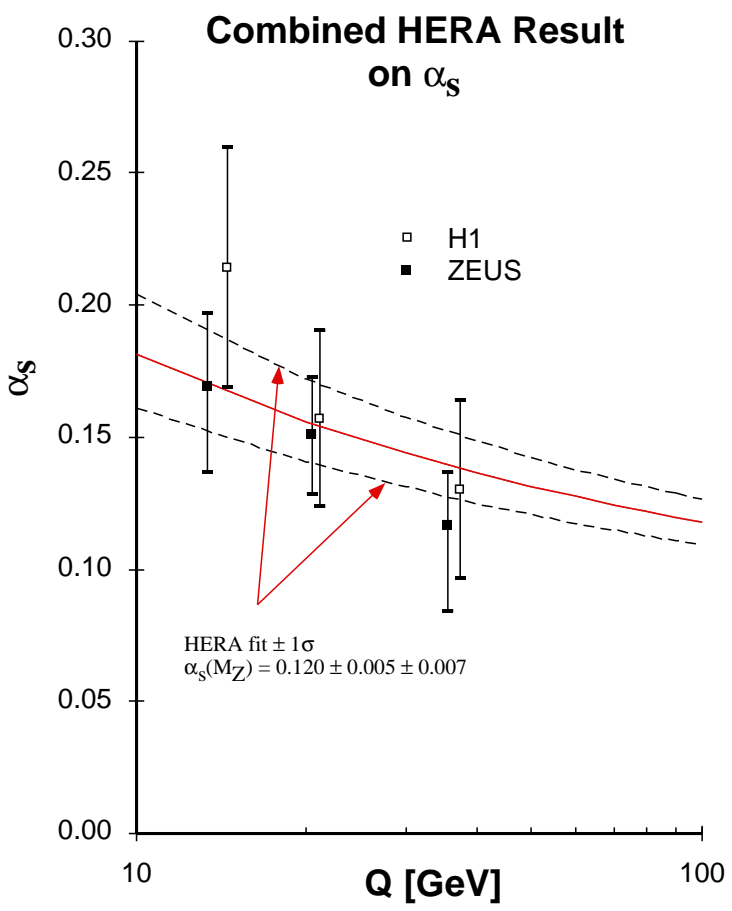

Figure 4. Measurements of $\alpha_{\mathrm{s}}\left(Q^{2}\right)$ from jet rates at HERA. The curves are the results of a QCD fit to the data (compilation from 23]).

functions. QCD predictions for $(1+1)$ and $(2+1)$ jet events which are complete to $\mathcal{O}\left(\alpha_{\mathrm{s}}^{2}\right)[22]$ are therefore used to extract $\alpha_{\mathrm{s}}$ for different bins of $Q^{2}$.

The results are compiled in Fig. 4 [23]. Although the overall uncertainties, both statistical as well as systematic, are still rather large in these measurements, the general trend of a coupling which decreases with increasing $Q$ can clearly be seen. The lines in Fig. 4 indicate the results of a QCD fit through the measurements, which extrapolates to $\alpha_{\mathrm{s}}\left(M_{\mathrm{Z}^{0}}\right)=0.120 \pm 0.005 \pm 0.007$.

In another study of the HERA data [24], $\alpha_{\mathrm{s}}$ is determined from the proton structure function $\mathrm{F}_{2}^{p}\left(x, Q^{2}\right)$ at small $x$ and $Q^{2}<100 \mathrm{GeV}^{2} . F_{2}^{p}$ is computed in next-to-leading order in $\alpha_{\mathrm{s}}$, including summations of all leading and subleading logarithms of $Q^{2}$ and $1 / x$. In that study it is demonstrated that the structure function data of $\mathrm{H} 1$ and of ZEUS exhibit double logarithmic scaling in both $x$ and $Q^{2}$, which is regarded as direct evi- 


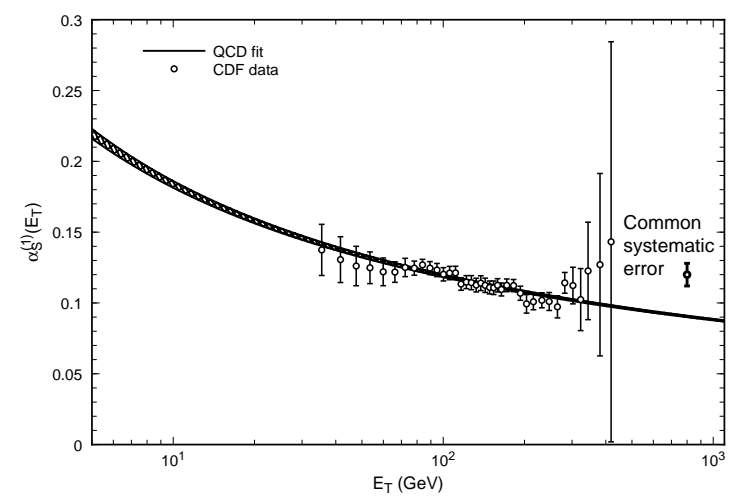

Figure 5. Values of $\alpha_{\mathrm{S}}\left(E_{T}\right)$ extracted from the onejet inclusive jet cross sections from $\mathrm{CDF}$ as a function of the the jet transverse energy $E_{T}$, together with the QCD expectations based on NLO perturbation theory and the MRSA' particle density function (from 25).

dence for the running $\alpha_{\mathrm{s}}$ 24]. A QCD fit to these data finally gives $\alpha_{\mathrm{s}}\left(M_{\mathrm{Z}^{0}}\right)=0.120 \pm 0.005 \pm 0.009$, where the first error is experimental and the second theoretical. This is in good agreement with the result from jets described above, and also with the world average of $\alpha_{\mathrm{s}}\left(M_{\mathrm{Z}^{0}}\right)$, see section 7 .

\section{JETS IN HADRON COLLISIONS}

Similarly as in deep inelastic lepton-nucleon scattering, hadron colliders provide the opportunity to simultaneously probe QCD in a wide range of momentum transfers $Q$. In a recent study [25] based on the one-jet inclusive transverse energy $\left(E_{T}\right)$ distribution measured at the Tevatron [26], values of $\alpha_{\mathrm{s}}\left(Q \equiv E_{T}\right)$ are determined over a wide range of energies, $E_{T}=30$ to $500 \mathrm{GeV}$.

The results of this study are shown in Fig. 5 . The values of $\alpha_{\mathrm{s}}\left(E_{T}\right)$ are seen to decrease with increasing $E_{T}$, in good agreement with the QCD expectations of a running coupling strength (shaded area). A simultaneous QCD fit to these data results in $\alpha_{\mathrm{s}}\left(M_{\mathrm{Z}^{0}}\right)=0.121 \pm 0.001$ (stat.) \pm 0.008 (syst.) \pm 0.005 (theor.), which is in excellent agreement with other measurements, see section 7 .

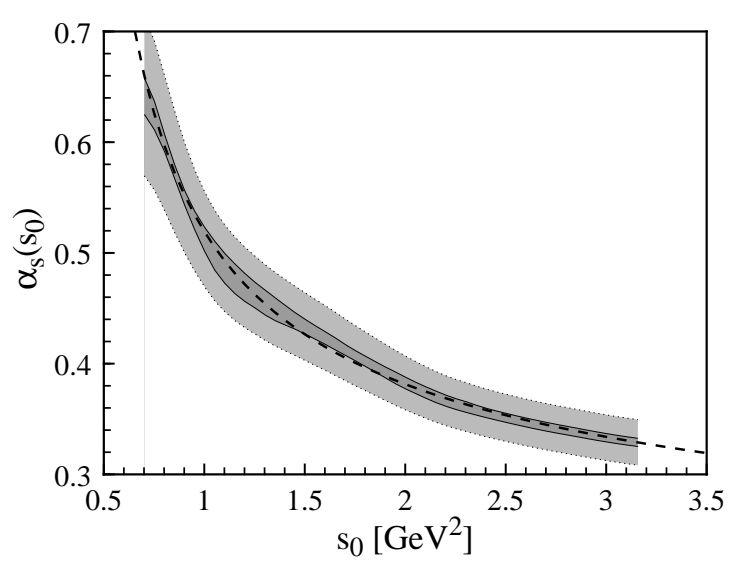

Figure 6. Values of $\alpha_{\mathrm{s}}\left(s_{0}\right)$ from the data on $R_{\tau}\left(s_{0}\right)$. The inner band represents experimental, the outer band the sum of experimental and theoretical uncertainties. The dashed line shows the running coupling constant in $\mathcal{O}\left(\alpha_{\mathrm{s}}^{3}\right)$ QCD. (From [28])

\section{RUNNING $\alpha_{\mathrm{S}}$ FROM $\tau$ DECAYS}

Measurements of the ratio of the hadronic and leptonic branching fractions of the $\tau$ lepton, $R_{\tau}$, have provided precise values of $\alpha_{\mathrm{s}}$ at the energy scale of the $\tau$-mass, $Q \equiv M_{\tau}=1.777 \mathrm{GeV}$, see e.g. 13, 27 and references quoted therein. Recently, a new test of the energy dependence of $\alpha_{\mathrm{s}}$ was proposed [28], based on the $\tau$ decay rate into hadrons of invariant mass squared $s$ smaller than a threshold value $s_{0}$ :

$$
\begin{aligned}
R_{\tau}\left(s_{0}\right) & =\frac{\Gamma\left(\tau \rightarrow \nu_{\tau}+\text { hadrons } ; s<s_{0}\right)}{\Gamma\left(\tau \rightarrow \nu_{\tau} e \overline{\overline{\nu_{e}}}\right)} \\
& =\int_{0}^{s_{0}} \mathrm{~d} s \frac{\mathrm{d} R_{\tau}(s)}{\mathrm{d} s} .
\end{aligned}
$$

The running coupling constant $\alpha_{\mathrm{s}}\left(s_{0}\right)$ is extracted from the inclusive hadronic spectrum $\mathrm{d} R_{\tau}(s) / \mathrm{d} s$ measured by ALEPH and CLEO [27,29], in the low energy region $0.7 \mathrm{GeV}^{2}<s_{0}<M_{\tau}^{2}$ where $\alpha_{\mathrm{s}}$ is expected to change by almost a factor of two.

Theoretical predictions for $R_{\tau}\left(s_{0}\right)$ include perturbative terms which are complete to $\mathcal{O}\left(\alpha_{\mathrm{s}}^{3}\right)$ as well as estimates of nonperturbative contributions using the operator product expansion 31.32]. Assuming global parton-hadron duality, $\alpha_{\mathrm{s}}$ can thus be determined from each measured value of $R_{\tau}\left(s_{0}\right)$. 
The results of the study of ref. 28 are shown in Fig. 6. Since values of $\alpha_{\mathrm{s}}$ extracted from $R_{\tau}\left(s_{0}\right)$ are correlated with each other, the fit results are displayed as a band. The dashed curve shows the QCD expectation of the running coupling constant calculated in $\mathcal{O}\left(\alpha_{\mathrm{s}}^{3}\right)$, normalised to the data at $s_{0}=M_{\tau}^{2}$.

The observed energy dependence is in excellent agreement with the QCD prediction of the running $\alpha_{\mathrm{s}}$ in $\mathcal{O}\left(\alpha_{\mathrm{s}}^{3}\right)$. In addition, the data show a distinct preference for the 3-loop $\beta$-function (Eq. 2), compared to the leading order (1-loop) one [28]. The overall value for $\alpha_{\mathrm{s}}$, including estimates of higher order perturbative uncertainties, results in $\alpha_{\mathrm{s}}\left(M_{\tau}\right)=0.33 \pm 0.03$ or, equivalently, in $\alpha_{\mathrm{s}}\left(M_{\mathrm{Z}^{0}}\right)=0.119 \pm 0.004$.

\section{WOLRD SUMMARY OF $\alpha_{\mathrm{s}}$}

Having discussed the current tests of asymptotic freedom from measurements based on single observables like jet rates, jet- $E_{T}$-spectra and $\tau$ decays, the overall summary of all available $\alpha_{\mathrm{s}}$ determinations remains to be updated (see e.g. 8,9, 13,30 for previous reviews).

An update of the summary given in [13] is presented in Table 1. Graphical presentations of the running coupling $\alpha_{\mathrm{s}}(Q)$ and of the results extrapolated to $\alpha_{\mathrm{s}}\left(M_{\mathrm{Z}^{0}}\right)$, using the 3-loop expansion (Eq. 2) and treating heavy flavour thresholds according to ref. [12], are given in Figs. 7 and 8, respectively. The most recent changes and additions are discussed in the following subsections; see 13] for comparison.

\section{1. $\alpha_{\mathrm{s}}$ from Sum Rules}

The results from the Gross-Llewellyn-Smith and the Bjorken sum rules [33, 34] have been retained; a preliminary update of the Bjorken sum rule result from the CCFR collaboration (see e.g. [35]) exists but is not included here because the final publication is still missing.

\section{2. $\alpha_{\mathrm{s}}$ from $\tau$ Decays}

In the previous report [13], $\alpha_{\mathrm{s}}$ from $\tau$ decays was obtained from a compilation of measurements of the ratio of the hadronic to the leptonic $\tau$ branching ratios, $R_{\tau}$. Meanwhile, several new measurements of this quantity became available

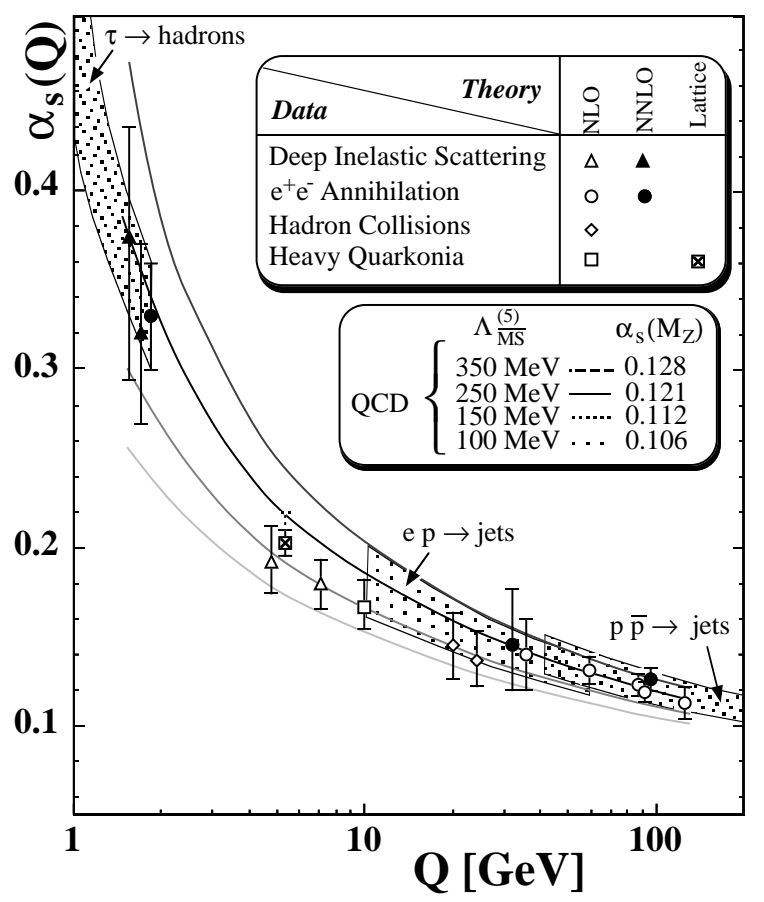

Figure 7. A Summary of measurements of $\alpha_{\mathrm{s}}$, compared with QCD expectations for four different values of $\Lambda_{\overline{M S}}$ which are given for $N_{f}=5$ quark flavours (relation between $\alpha_{\mathrm{s}}$ and $\Lambda_{\overline{M S}}$ in $\mathcal{O}\left(\alpha_{\mathrm{s}}^{3}\right)$ ).

(see [27,36] and references quoted therein). Instead of deriving an updated value of $\alpha_{\mathrm{s}}$ from $R_{\tau}$, the results from the study described in section 6 , namely from the hadronic invariant mass distribution of $\tau$ decays, $R_{\tau}\left(s_{0}\right)$, is taken. This result is identical to the one derived from a recent evaluation of $\alpha_{\mathrm{s}}$ and its overall uncertainty from $R_{\tau}$ [38]: $\alpha_{\mathrm{s}}\left(M_{\tau}\right)=0.33 \pm 0.03$.

\section{3. $\alpha_{\mathrm{S}}$ from Deep Inelastic Scattering}

In addition to the earlier results from fixed target experiments [39,40], the new values of $\alpha_{\mathrm{s}}$ from HERA, discussed in Sect. 4, are included.

\section{4. $\alpha_{\mathrm{s}}$ from Lattice QCD}

The values of $\alpha_{\mathrm{s}}\left(M_{\mathrm{Z}^{0}}\right)$ from lattice QCD calculations, based on measurements of heavy quarkonia mass spectra, slowly but gradually increased during the past few years. These changes are mainly due to the availability of unquenched cal- 


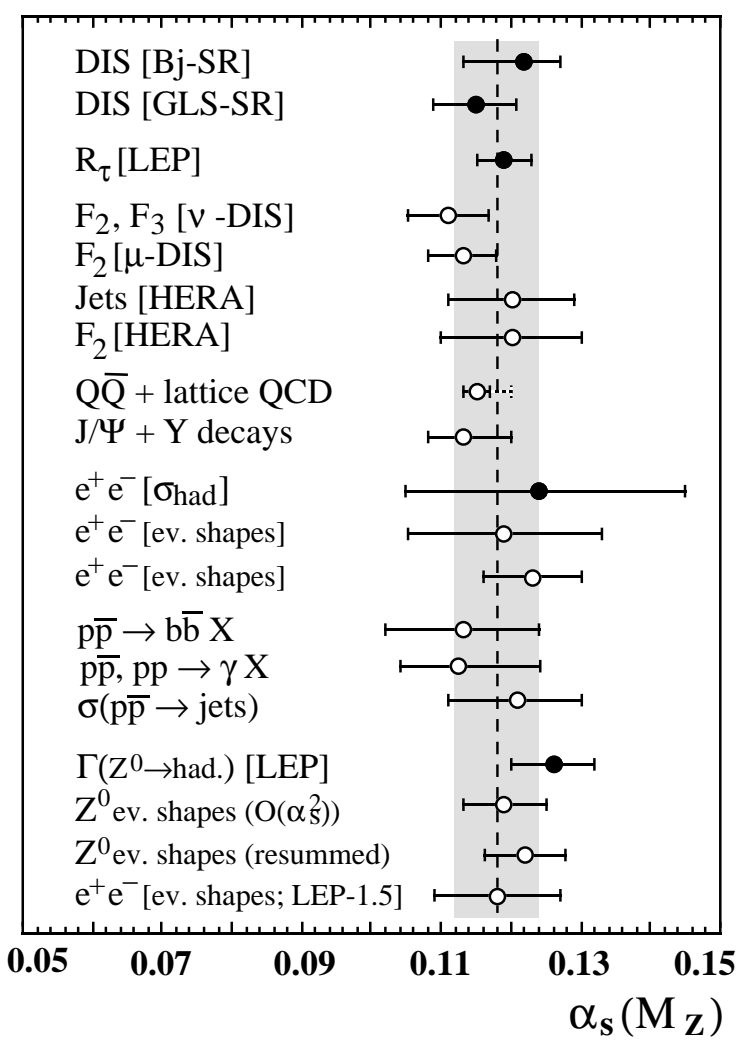

Figure 8. A Summary of measurements of $\alpha_{\mathrm{S}}\left(M_{\mathrm{Z}^{0}}\right)$, as listed in Table 1. Filled symbols are derived using $\mathcal{O}\left(\alpha_{\mathrm{s}}^{3}\right)$ QCD; open symbols are in $\mathcal{O}\left(\alpha_{\mathrm{s}}^{2}\right)$ or based on lattice calculations.

culations (i.e. including dynamical light quark flavours) and to more refined procedures to convert the lattice coupling to the running coupling of perturbative QCD. A recent summary of these results gives $\alpha_{\mathrm{S}}\left(M_{\mathrm{Z}^{0}}\right)=0.115 \pm 0.002$ 41, which is taken over for this review. There are, however, unpublished reports which result in $\alpha_{\mathrm{s}}\left(M_{\mathrm{Z}^{0}}\right)=$ $0.118 \pm 0.002$, see e.g. [42].

\section{5. $\alpha_{\mathrm{s}}$ from Hadron Collsions}

The previous, preliminary determination of $\alpha_{\mathrm{s}}$ from a measurement of the $b \bar{b}$ cross section was updated and finally published in 43 .

Results on $\alpha_{\mathrm{s}}$ from $\mathrm{p} \overline{\mathrm{p}} \rightarrow \mathrm{W}$ jets 44, 45, which were considered in previous compilations are no longer included since the QCD calculations on which they are based are not complete to next- to-leading order. A recent study of this process from D0 reports that calculations which are complete to NLO do not provide a reasonable fit of the data 46.

The result on $\alpha_{\mathrm{s}}$ from the one-jet inclusive $E_{t^{-}}$ distribution, as discussed in Section 5, is a new entry in Table 1.

\section{6. $\alpha_{\mathrm{s}}$ from the $\mathrm{Z}^{0}$ Line Shape}

The value of $\alpha_{\mathrm{s}}\left(M_{\mathrm{Z}^{0}}\right)$ derived from the hadronic width of the $\mathrm{Z}^{0}$ boson was continuously updated during the past years, according to the increasing data statistics of the four LEP experiments. Not all of these updates were published in journals, however they are documented as CERN preprints which are commonly available. In this review, the result which was documented last before this conference is taken, $\alpha_{\mathrm{s}}\left(M_{\mathrm{Z}^{0}}\right)=0.126 \pm 0.006$. 447.

\section{7. $\alpha_{\mathrm{s}}$ from Event Shapes at LEP-1.5}

Three of the LEP experiments have published determinations of $\alpha_{\mathrm{s}}$ from the data taken at $\mathrm{e}^{+} \mathrm{e}^{-}$ c.m. energies between 130 and $136 \mathrm{GeV}$ 48, 49, 19. Each experiment collected about $5 p b^{-1}$ of data, corresponding to only about 300 nonradiative hadronic events per experiment. Due to the large statistical uncertainty of each experiment, only the combined value of $\alpha_{\mathrm{s}}$ from LEP1.5 can provide a meaningful test of the running coupling.

The results of $\alpha_{\mathrm{s}}$ from jet rates and from hadronic event shapes at LEP-1.5 are summarised in Table 2. There is good agreement between the experiments, within the statistical uncertainties. The average result is $\alpha_{\mathrm{s}}(133 \mathrm{GeV})=0.112 \pm 0.009$ or, equivalently, $\alpha_{\mathrm{s}}\left(M_{\mathrm{Z}^{0}}\right)=0.118 \pm 0.009$, which is compatible, within the experimental errors, with the value which was directly obtained at the $\mathrm{Z}^{0}$ resonance, $\alpha_{\mathrm{s}}\left(M_{\mathrm{Z}^{0}}\right)=0.121 \pm 0.006$.

\subsection{World Average of $\alpha_{\mathrm{s}}\left(M_{\mathrm{Z}^{0}}\right)$}

Averaging the values of $\alpha_{\mathrm{s}}\left(M_{\mathrm{Z}^{0}}\right)$ from Table 1, either unweigthed or weigthed by the inverse square of their errors, gives $\overline{\alpha_{\mathrm{s}}}\left(M_{\mathrm{Z}^{0}}\right)=0.118$ in both cases. This value has been remarkably sta-

${ }^{\dagger}$ The result which is based on lattice QCD is not included when computing the weigthed average; see Section 7.4 for justification. 
ble during the past few years, see e.g. $8,8,9,13,30$ for previous reviews. From Fig. 8 it can be seen that all results of $\alpha_{\mathrm{s}}$ are compatible with this world average, within the errors assigned to the measurements.

The errors of most $\alpha_{\mathrm{s}}$ results are dominated by theoretical uncertainties, which are estimated using a variety of different methods and definitions. The significance of the quoted errors is largely unknown; they are neither gaussian nor are the correlations between different measurements known. A "correct" calculation of the overall uncertainty of $\overline{\alpha_{\mathrm{s}}}\left(M_{\mathrm{Z}^{0}}\right)$ is therefore not possible.

Some methods were proposed to compute the overall error from the individual ones, either by rescaling the latter or by constructing an ad-hoc correlation matrix such that the overall $\chi^{2}$ deviation from the mean value is equal to the number of degrees of freedom (i.e. to $n-1$, where $n$ is the number of individual measurements) [10,50]. If applied to the results listed in Table 1, these methods suggest that $\Delta \overline{\alpha_{\mathrm{S}}}\left(M_{\mathrm{Z}^{0}}\right) \sim 0.003 \ldots 0.005$.

Since most of the errors listed in Table 1 are not gaussian but rather indicate probability distributions of rectangular shape (however still with unknown correlations between each other), a more pragmatic and conservative estimate of the overall uncertainty of $\overline{\alpha_{\mathrm{S}}}\left(M_{\mathrm{Z}^{0}}\right)$ is therefore applied: counting the relative number of entries in Table 1 whose central values are within $\pm \Delta \overline{\alpha_{\mathrm{s}}}$ of $\overline{\alpha_{\mathrm{s}}}\left(M_{\mathrm{Z}^{0}}\right)=0.118$, one gets about $45 \%$ for $\Delta \overline{\alpha_{\mathrm{s}}}=$ $0.003,60 \%$ for $0.004,75 \%$ for $0.005,90 \%$ for $0.006,95 \%$ for 0.008 and $100 \%$ for 0.008 . A $90 \%$ "confidence level" seems to be a reasonable and safe estimate for $\Delta \overline{\alpha_{\mathrm{s}}}$, such that the world average is quoted to be

$$
\overline{\alpha_{\mathrm{s}}}\left(M_{\mathrm{Z}^{0}}\right)=0.118 \pm 0.006,
$$

which corresponds, in $\mathcal{O}\left(\alpha_{\mathrm{s}}^{3}\right)$ and for $N_{f}=5$ or 4 flavours, to

$$
\Lambda \frac{(5)}{M S}=210_{-65}^{+80} \mathrm{MeV}, \text { or } \Lambda \frac{(4)}{M S}=295_{-80}^{+95} \mathrm{MeV} .
$$

The world average is indicated by the vertical line and the shaded area in Fig. 8.

7.9. Systematic Differences in $\alpha_{\mathrm{S}}\left(M_{\mathrm{Z}^{0}}\right)$ ?

In previous reviews the observation was made that measurements which are obtained at energy scales of $5 \mathrm{GeV}<Q<20 \mathrm{GeV}$ are systematically low, corresponding to $\alpha_{\mathrm{S}}\left(M_{\mathrm{Z}^{0}}\right) \approx 0.112$, while at $Q \approx M_{\tau}$ and $Q \geq 30 \mathrm{GeV}$ the results tend to be higher, $\alpha_{\mathrm{s}}\left(M_{\mathrm{Z}^{0}}\right) \approx 0.120$ and 0.122 , respectively. Speculations about the origin of these differences include the existence of a light, neutral, coloured object of spin 1/2 (e.g. a gluino), a possible dependence on the scattering process $\left(\mathrm{e}^{+} \mathrm{e}^{-}\right.$annihilation or deep inelastic scattering) or effects of quark masses which are not included in the current higher order QCD calculations [13].

In general, these systematic but hardly conclusive differences are still visible in this summary, see Table 1 and Figure 8. However, the most recent results of $\alpha_{\mathrm{s}}$ from deep inelastic scattering at HERA and from jet production in hadron collisions underline the tendency towards higher values of $\alpha_{\mathrm{s}}\left(M_{\mathrm{Z}^{0}}\right)$, in agreement with those from $\mathrm{e}^{+} \mathrm{e}^{-}$annihilation, and the detailed studies of hadronic $\tau$-decays provide consistent results, too. Therefore the hyptheses of a process dependence of $\alpha_{\mathrm{s}}$ or of the existence of light gluinos are not very likely to explain the suspected differences. The most probable origin of those, if significant at all, could be the absence of heavy quark mass effects in the current QCD calculations, which would affect the results from data with energies close to the quark thresholds most.

Next-to-leading order calculations including quark mass effects are currently being worked on. This, together with the ongoing efforts to determine $\alpha_{\mathrm{s}}$ from the yet increasing amount of data from various processes, has the potential to decrease systematic uncertainties and to resolve the cause of the differences which are still being observed.

\section{Conclusion}

Asymptotic freedom, which is the key feature of the theory of strong interactions, has been successfully tested in various experimental studies. Perhaps the most intuitive and direct method, the study of the energy dependence of 3-jet event production rates in $\mathrm{e}^{+} \mathrm{e}^{-}$annihilation, $R_{3}$, began to provide evidence for the running of $\alpha_{\mathrm{s}}$ already in 1988. These studies, carried out by various experiments in a large range of c.m. energies, are 
based on the JADE-E0 jet algorithm for which, at constant jet resolution, hadronisation corrections are small enough such that $R_{3}$ is directly proportional to $\alpha_{\mathrm{s}}$. With the availability of the LEP data the evidence developed into a proof of asymptotic freedom, demonstrating that $\alpha_{\mathrm{s}}$ decreases with increasing energy, as predicted by QCD.

Further significant tests became available in the past two years: jet rates in ep-collisions at HERA and 1-jet inclusive transverse energy distributions in hadron collisions, measured by single experiments in large regions of the energy scale, provide the possibility to determine the energy dependence of $\alpha_{\mathrm{s}}$ while minimising systematic point-to-point uncertainties. A new analysis based on invariant mass distributions of hadronic $\tau$-decays demonstrates the running of $\alpha_{\mathrm{s}}$ in the energy range of $0.7 \mathrm{GeV}^{2}<Q^{2}<M_{\tau}^{2}$, where the coupling changes by almost a factor of two.

In addition to these dedicated tests, the world summary of measurements of $\alpha_{\mathrm{s}}$, in the energy range of $M_{\tau} \leq Q \leq 133 \mathrm{GeV}$, provides compelling evidence for asymptotic freedom. Within their assigned uncertainties, all measurements are compatible with the QCD expectation of a running $\alpha_{\mathrm{s}}$.

Extrapolated to a common energy scale, using Equations 1 and 2 and treating quark flavour thresholds as described in 12,10], the measurements of $\alpha_{\mathrm{s}}$ average to

$$
\alpha_{\mathrm{s}}\left(M_{\mathrm{Z}^{0}}\right)=0.118 \pm 0.006 .
$$

The overall uncertainty of 0.006 corresponds to a simple estimate of a "90\% confidence level", derived from the scatter of the individual results.

Acknowledgements. It is a pleasure to thank S. Narison for providing the possibility to present this review at this well organised and informative conference. I am grateful to E. Elsen, W. Giele, and M. Neubert for providing and authorising the use of Figures 4, 5 and 6, and to W. Bernreuther for many interesting discussions.

\section{REFERENCES}

1. H. Fritzsch, M. Gell-Mann and H. Leutwyler, Phys. Lett. B47 (1973) 365; D.J. Gross, F. Wilczek, Phys. Rev. D8 (1973) 3633; D.J. Gross, F. Wilczek, Phys. Rev. D9 (1974) 980; H.D. Politzer, Phys. Rep. 14 (1974) 129.

2. D.J. Gross, F. Wilczek, Phys. Rev. Lett. 30 (1973) 1343; H.D. Politzer, Phys. Rev. Lett. 30 (1973) 1346.

3. G. Hanson et al., Phys. Rev. Lett 35 (1975) 1609.

4. R. Brandelik et al., TASSO collab., Phys. Lett. B86 (1979) 243; D.P. Barber et al., MARK J collab., Phys. Rev. Lett 43 (1979) 830; Ch. Berger et al., PLUTO collab., Phys. Lett. B86 (1979) 418; W. Bartel et al., JADE collab., Phys. Lett. B91 (1980) 142.

5. D.P. Barber et al., MARK J collab., Phys. Lett B89 (1979) 139.

6. W. Bartel et al., JADE collab., Phys. Lett. B119 (1982) 239.

7. S. Bethke et al., JADE collab., Phys. Lett. B213 (1988), 235.

8. G. Altarelli, Ann. Rev. Nucl. Part. Sci 39 (1989) 357.

9. S. Bethke and S. Catani, Proc. of the XXVII h Rencontre de Moriond, Les Arcs, France, 1992; CERN-TH.6484/92.

10. Review of Particle Properties, Phys. Rev. D54 (1996).

11. W.A. Bardeen et al., Phys. Rev. D18 (1978) 3998.

12. W. Bernreuther and W. Wetzel, Nucl. Phys. B197 (1982) 228.

13. S. Bethke, Proc. of the QCD'94, Montpellier 1994, Nucl. Phys. B (Proc. Suppl.) 39B,C (1995) 198.

14. S. Bethke, Z. Kunszt, D.E. Soper, W.J. Stirling, Nucl. Phys. B370 (1992) 310.

15. R.K. Ellis, D.A. Ross, A.E. Terrano, Nucl. Phys. B178 (1981) 421.

16. T. Sjöstrand, CERN-TH-7112-93 (Feb. 1994).

17. S. Bethke, Proc. of the $42^{\text {nd }}$ Scottish Universities Summer School in Physics, St. Andrews 1993; HD-PY 93/7.

18. W. Braunschweig et al., TASSO collab., Phys. Lett. B214 (1988) 286; I. Park et al., AMY collab., Phys. Rev. Lett 62 (1989) 1713; K. Abe et al., VENUS collab., Phys. Lett. B240 
(1990) 232; M.Z. Akrawy et al., OPAL collab., Z. Phys. C49 (1991) 375; D. Decamp et al., Phys. Lett. B255 (1991) 623; P. Abreu et al., DELPHI collab., Phys. Lett. B247 (1990) 167; B. Adeva et al., L3 collab., Phys. Lett. B248 (1990) 464.

19. G. Alexander et al., OPAL collab., CERNPPE/96-047, accpt. by Z. Phys. C.

20. T. Ahmed et al., H1 collab., Phys. Lett B346 (1995) 415.

21. M. Derrick et al., ZEUS collab., Phys. Lett. B363 (1995) 201.

22. D. Graudenz, Phys. Rev. D49 (1994) 3291.

23. E. Elsen in Proc. Int. Europhysics Conf on High Energy Physics, Brussels (1995), Eds. J. Lemonne, C. Vander Velde, F. Verbeure, World Scientific.

24. R. Ball, S. Forte, Phys. Lett. B358 (1995) 365.

25. W.T. Giele, E.W.N. Glover, J. Yu, Phys. Rev. D53 (1996) 120.

26. F. Abe et al., CDF collab., Phys. Rev. Lett 68 (1992) 1104.

27. L. Duflot, Proc. of the QCD'94, Montpellier 1994, Nucl. Phys. B (Proc. Suppl.) 39B,C (1995); Proc. of the TAU'94, Montreux 1994, Nucl. Phys. B (Proc. Suppl.) 40 (1995) 37.

28. M. Girone, M. Neubert, Phys. Rev Lett. 76 (1996) 3061.

29. T. Coan et al., CLEO collab., Phys. Lett. B356 (1995) 580.

30. S. Bethke, Proc. of the $X X X^{\text {th }}$ Rencontre de Moriond, Les Arcs, France, 1995; PITHA 95/14.

31. M.A. Shifman, A.I. Vainsthein and V.I. Zakharov, Nucl. Phys. B147 (1979) 385 and 448.

32. E. Braaten, S. Narison and A. Pich, Nucl. Phys. B373 (1992) 581.

33. J. Ellis and M. Karliner, Phys. Lett. B341 (1995) 397.

34. J. Chyla and A.L. Kataev, Phys. Lett. B297 (1992) 385.

35. D. Harris, CCFR collab., Proc. $X X X^{\text {th }}$ Rencontre de Moriond, Les Arcs, France, March 1995.

36. G. Alexander et al., OPAL collab. Phys. Lett. B369 (1996) 163.

37. S. Narison, Proc. of the TAU'94, Montreux 1994, Nucl. Phys. B (Proc. Suppl.) 40 (1995)
198.

38. S. Narison, Phys. Lett. B361 (1995) 121.

39. P.Z. Quintas et al., CCFR collab., Phys. Rev. Lett. 71 (1993) 1307.

40. M. Virchaux and A. Milsztajn, Phys. Lett. B274 (1992) 221.

41. C.T.H. Davies et al., Phys. Lett. B345 (1995) 42.

42. G.P. Lepage, hep-lat/9607076 (1996).

43. C. Albajar et al., UA1 collab., Phys. Lett. B369 (1996) 46.

44. J. Alitti et al., UA2 collab., Phys. Lett. B263 (1991) 563.

45. M. Lindgren et al., UA1 collab., Phys. Rev. D45 (1992) 3038.

46. S. Abachi et al., D0 collab., Phys. Rev. Lett. 75 (1995) 3226.

47. The LEP Electroweak Working Group, LEPEWWG/96-01 (March 1996).

48. M. Acciarri et al., L3 collab., Phys. Lett. B 371 (1996) 137-148.

49. D. Buskulic et al., ALEPH collab., CERN PPE/96-043, subm. to Z. Phys. C.

50. M. Schmelling, Physica Scripta 51 (1995) 676.

51. M. Kobel, Proc. of the XXVII Rencontres de Moriond, Les Arcs, France, 1992.

52. D. Haidt, in Directions in High Energy Physics Vol 14, Precision Tests of the Standard Electroweak Model, ed. Langacker P, World Scientific Company, 1993.

53. S. Bethke, Proc. of the Workshop on the Standard Model at the Energy of Present and Future Accelerators, June 1989, Budapest; preprint LBL-28112 (1989).

54. Y. Ohnishi et al. TOPAZ collab., Phys. Lett. B 313 (1993) 475.

55. G. Ballocchi et al., UA6 collab., Phys. Lett. B 317 (1993) 250. 


\begin{tabular}{|c|c|c|c|c|c|c|c|}
\hline & & $\mathrm{Q}$ & & & $\Delta \alpha_{\mathrm{s}}$ & $\overline{\left.M_{Z^{0}}\right)}$ & \\
\hline Process & Ref. & {$[\mathrm{GeV}]$} & $\alpha_{s}(Q)$ & $\alpha_{\mathrm{s}}\left(M_{\mathrm{Z}^{0}}\right)$ & exp. & theor. & Theory \\
\hline DIS $[\nu ; \mathrm{Bj}-\mathrm{SR}]$ & 33 & 1.58 & $0.375_{-0.081}^{+0.062}$ & $0.122+0.005$ & - & - & NNLO \\
\hline DIS $[\nu ;$ GLS-SR $]$ & 34 & 1.73 & $0.32 \pm 0.05$ & $0.115 \pm 0.006$ & 0.005 & 0.003 & NNLO \\
\hline$\tau$-decays & 38,28 & 1.78 & $0.330 \pm 0.030$ & $0.119 \pm 0.004$ & 0.001 & 0.004 & NNLO \\
\hline $\operatorname{DIS}\left[\nu ; \mathrm{F}_{2}\right.$ and $\left.\mathrm{F}_{3}\right]$ & 39 & 5.0 & $0.193_{-0.018}^{+0.019}$ & $0.111 \pm 0.006$ & 0.004 & 0.004 & NLO \\
\hline $\operatorname{DIS}\left[\mu ; \mathrm{F}_{2}\right]$ & 40 & 7.1 & $0.180 \pm 0.014$ & $0.113 \pm 0.005$ & 0.003 & 0.004 & NLO \\
\hline DIS [HERA; jets] & 20 & $10-60$ & & $0.120 \pm 0.009$ & 0.005 & 0.007 & NLO \\
\hline DIS [HERA; $\mathrm{F}_{2}$ ] & 24 & $2-10$ & & $0.120 \pm 0.010$ & 0.005 & 0.009 & NLO \\
\hline $\mathrm{Q} \overline{\mathrm{Q}}$ states & 41 & 5.0 & $0.203 \pm 0.007$ & $0.115 \pm 0.002$ & 0.000 & 0.002 & LGT \\
\hline$J / \Psi+\Upsilon$ decays & 51 & 10.0 & $0.167 \stackrel{+0.015}{+0.011}$ & $0.113+0.007$ & 0.001 & $\begin{array}{l}+0.007 \\
-0.005\end{array}$ & NLO \\
\hline $\mathrm{e}^{+} \mathrm{e}^{-}\left[\sigma_{\mathrm{had}}\right]$ & 52 & 34.0 & $0.146+0.031$ & $0.124+0.021$ & $\begin{array}{l}+0.021 \\
-0.019\end{array}$ & - & NLO \\
\hline $\mathrm{e}^{+} \mathrm{e}^{-}$[ev. shapes $]$ & 53 & 35.0 & $0.14 \pm 0.02$ & $0.119 \pm 0.014$ & - & - & NLO \\
\hline $\mathrm{e}^{+} \mathrm{e}^{-}$[ev. shapes] & 54 & 58.0 & $0.132 \pm 0.008$ & $0.123 \pm 0.007$ & 0.003 & 0.007 & resum. \\
\hline$p \bar{p} \rightarrow \mathrm{b} \overline{\mathrm{b} X}$ & 43 & 20.0 & $0.145_{-0.019}^{+0.018}$ & $0.113 \pm 0.011$ & $\begin{array}{l}+0.007 \\
-0.006\end{array}$ & $\begin{array}{l}+0.008 \\
+0.009\end{array}$ & NLO \\
\hline $\mathrm{p} \overline{\mathrm{p}}, \mathrm{pp} \rightarrow \gamma \mathrm{X}$ & 55 & 24.2 & $0.137_{-0.014}^{+0.017}$ & $0.112+0.012$ & 0.006 & $\begin{array}{l}-0.010 \\
+0.005 \\
-0.05\end{array}$ & NLO \\
\hline$\sigma(\mathrm{p} \overline{\mathrm{p}} \rightarrow$ jets $)$ & 25 & $30-500$ & & $0.121 \pm 0.009$ & 0.001 & 0.009 & NLO \\
\hline $\mathrm{e}^{+} \mathrm{e}^{-} \rightarrow \mathrm{Z}^{0}:$ & & & & & & & \\
\hline$\Gamma\left(\mathrm{Z}^{0} \rightarrow\right.$ had. $)$ & 47 & 91.2 & $0.126 \pm 0.006$ & $0.126 \pm 0.006$ & 0.005 & 0.003 & NNLO \\
\hline had. event shapes & 13 & 91.2 & $0.119 \pm 0.006$ & $0.119 \pm 0.006$ & 0.001 & 0.006 & NLO \\
\hline had. event shapes & 13 & 91.2 & $0.122 \pm 0.006$ & $0.122 \pm 0.006$ & 0.001 & 0.006 & resum. \\
\hline $\mathrm{e}^{+} \mathrm{e}^{-}[$ev. shapes $]$ & Tab. 2 & 133.0 & $0.112 \pm 0.009$ & $0.118 \pm 0.009$ & 0.003 & 0.009 & resum. \\
\hline
\end{tabular}

Table 1. World summary of measurements of $\alpha_{\mathrm{s}}$. Abbreviations: DIS $=$ deep inelastic scattering; GLS-SR $=$ Gross-Llewellyn-Smith sum rules; Bj-SR = Bjorken sum rules; (N)NLO = (next-)next-to-leading order perturbation theory; LGT = lattice gauge theory; resum. = resummed next-to-leading order.

\begin{tabular}{|l|c|c|c||c|c|}
\hline Exp. & Ref. & $\alpha_{\mathrm{S}}(133 \mathrm{GeV})$ & $\rightarrow \alpha_{\mathrm{S}}\left(M_{\mathrm{Z}^{0}}\right)[\mathrm{LEP}-1.5]$ & $\alpha_{\mathrm{S}}\left(M_{\mathrm{Z}^{0}}\right)[$ LEP-I $]$ & $\# \sigma$ \\
\hline ALEPH & {$[49$} & $0.119 \pm 0.005 \pm 0.007$ & $0.126 \pm 0.006 \pm 0.008$ & $0.120 \pm 0.002 \pm 0.007$ & 0.95 \\
L3 & {$\left[\frac{48}{49}\right.$} & $0.107 \pm 0.005 \pm 0.006$ & $0.113 \pm 0.006 \pm 0.007$ & $0.125 \pm 0.003 \pm 0.008$ & 1.8 \\
OPAL & {$[19]$} & $0.110 \pm 0.005 \pm 0.009$ & $0.116 \pm 0.006 \pm 0.010$ & $0.120 \pm 0.002 \pm 0.006$ & 0.63 \\
\hline Average & & $0.112 \pm 0.003 \pm 0.008$ & $0.118 \pm 0.003 \pm 0.009$ & $0.122 \pm 0.001 \pm 0.006$ & 0.95 \\
\hline
\end{tabular}

Table 2. Summary of measurements of $\alpha_{\mathrm{s}}$ at LEP-1.5 $\left(<E_{c m}>=133 \mathrm{GeV}\right)$. The first errors are experimental, the second theoretical. The last two columns give the results of $\alpha_{\mathrm{s}}\left(M_{\mathrm{Z}^{0}}\right)$ previously obtained at the $\mathrm{Z}^{0}$ resonance (LEP-I) and the number of standard deviations between the LEP-I and LEP-1.5 results, respectively, taking only experimental errors into account. 